\title{
Viability Assessment of Chondrocytes Obtained from Flap Type Injuries in Patients with FAI
}

\author{
Tomas Amenabar ${ }^{1,2 *}$, Claudio Rafols ${ }^{1}$, Juan Monckeberg ${ }^{1}$, Jorge Vergara $^{1}$ \\ ${ }^{1}$ Clinica MEDS, Santiago, Chile. \\ ${ }^{2}$ Instituto Traumatológico, Santiago, Chile.
}

Received: October 18, 2016; Accepted: December 21, 2016; Published: February 7, 2017

*Corresponding author: Tomas Amenabar, Espoz 3355, apartment B202, Vitacura, Santiago, Chile, 7630685, Tel: +56991594716; E-mail: tomasamenabar@gmail.com

\section{Abstract}

Introduction: It is well known that FAI leads to articular cartilage damage and flap type lesions in the acetabulum. Currently the treatment of these lesions remains controversial and repairing techniques have been proposed. Our purpose is to determine the viability and duplication capabilities of flap's chondrocytes in FAI.

Methods: Prospective study. Inclusion criteria were FAI and chondral flap at the time of surgery. Group A consisted of 9 cartilage flaps obtained from 9 hips in 6 patients. Group Bconsisted of samples from the head-neck junction prior to femoral osteoplasty from 4 hips in 2 patients

The samples had mechanical and enzymatic digestion in a standardized way. For cellular viability assessment the Trypan Blue dye test was used and the count was done under light microscope.

For duplication analysis the cellular pellet was seeded in 96 well plates and kept for 10 days in Dulbeco's Modified Eagle's Medium under standardized conditions. To determine the growing curves the method used was MTT colorimetric assay3-(4,5-dimethylthiazol-2yl)-2,5-diphenyltetrazolium bromide).

Mann-Whitney test was used to compare the duplication times between Group A and B, a p value $<0.05$ was considered significant.

Results: All the samples from both groups showed chondrocyte viability with a mean cellular viability of $43 \%$.

The average duplication time was $58.5+19.5$ hours for Group Aand $58+15.1$ hours for Group B with no significant difference between them $(\mathrm{p}=0.85)$

Conclusion: Our study shows cellular viability and duplication of chondrocytes obtained from cartilage flap in FAI.

\section{Introduction}

During recent years we have seen an increase in the number of hip arthroscopies mainly due to a better understanding of femoro acetabular impingement (FAI) [1-3].

It is well known that the morphologic conflict present in FAI leads to articular cartilage damage, especially in the antero superior aspect of the acetabulum. The presence of cartilage damage has been shown to be a bad prognostic factor and it is believed that it could lead to early osteoarthritis [4-7].

The cartilage damage of the hip secondary to FAI occurs mainly in the chondro-labral junction and progresses from loss of fixation to the subchondral bone (ie wave sign) to cleavage tear with an obvious separation of the chondrolabral junction but no delamination, to macroscopic debonding of the articular cartilage from the acetabular bone (also known as delamination or flap). The end stage involves exposed bone in the acetabulum $[8,9]$.

Currently the treatment of flap type chondral injuries remains controversial. The preferred method is debridement associated with bone marrow stimulation techniques (ie microfracture or microdrilling) for fibrocartilage fill. On the other hand, repairing techniques such as chondral sutures or the use of fibrin adhesive have been proposed [10-12]

The purpose of this study was to determine the viability and duplication capabilities of chondrocytes obtained from flap type cartilage injuries in patients with FAI. Our hypothesis was that the acetabular chondral flap would show cellular viability and duplication capabilities in an in vitro setting.

\section{Methods}

\section{Patient inclusion and data collection}

Ethics board approval was obtained to perform a prospective study. Inclusion criteria consisted of all the patients who underwent hip arthroscopy due to FAI, had a chondral flap at the time of surgery and consented to participate in the study.

Exclusion criteria were history of previous hip surgery or hip injections.

All the surgeries were performed by the senior author, with vast experience in hip arthroscopy, and the surgery was done in a standardized way with the patient in a supine position and using the outside-inside approach. The acetabular chondral flap found was resected and the lesion micro fractured. Labral refixation and acetabular and/or femoral osteoplasty were performed as needed. 
From August 2014 to October 2014, 9 cartilage flaps were obtained from 9 hips in 6 patients (Group A). Also, from 4 hips in 2 patients' samples from the head-neck junction were obtained prior to femoral osteoplasty as a control group (Group B).

\section{Sample processing and analysis}

Cartilage flaps were excised, stored in a phosphate-buffered saline solution (PBS, Sigma-Aldrich $\AA^{\circledR}$ ) at $4^{\circ} \mathrm{C}$ and transferred to the laboratory. Once in the laboratory the samples were cut in 1-3 mm3 pieces on Petridish under a laminar flow hood. Then the samples were washed and re-suspended 3 times in $1 \mathrm{X}$ PBS (pH7.4). After decantation the supernatant was discarded and the cellular pellet was exposed to enzymatic digestion in Dulbeco's Modified Eagle's Medium (DMEM, Sigma-Aldrich $(\circledR)$ at $37^{\circ} \mathrm{C}$ for 12 hours. The enzymatic solution consisted of $0.1 \%$ de Collagenase II (Gibco/Life Technologies ®), 0,01\% of Hyaluronidase II (AppliChem $\AA$ ) and $0.015 \%$ Deoxyribonuclease I (AppliChem $\left.{ }^{\circledR}\right)$.

After enzymatic digestion samples were centrifuged at 200G for 5 minutes, the cellular pellet was washed twice with PBS and aliquots were obtained for cellular viability assessment using Trypan Blue Solution at $0.4 \%$ (Sigma-Aldrich $®$ ). Trypan Blue is a dye exclusion test and is based upon the concept that viable cells do not take up impermeable dyes (like Trypan Blue), but dead cells are permeable and take up the dye. The viability count was done under light microscope.

The pellet was seeded in DMEM containing $10 \%$ of fetal bovine serum in 96 well plates and incubated at 37으 $5 \% \mathrm{CO} 2$. To determine the growing curves the method used was MTT colorimetric assay (3-(4,5-dimethylthiazol-2-yl)-2,5diphenyltetrazolium bromide) (Sigma-Aldrich $\AA$ ). MTT is cleaved to formazan by enzymes of the endoplasmic reticulum. This bio reduction occurs in viable cells only; therefore, the amount of formazan dye formed directly correlates to the number of metabolically active cells in the culture. After solubilization, the formazan dye is quantitated using a spectrophotometer (EPOCH spectrophotometer, Biotek $®$ ). At the beginning of each culture a calibration curve was done

For 10 days 201 of $5 \mathrm{mg} / \mathrm{ml}$ of MTT solution (Gibco/Life Technologies $\AA$ ) was added to a plate and cultured at $37^{\circ} \mathrm{C}$ $5 \% \mathrm{CO}_{2}$ for 3-5 hours. After that Dimethyl Sulfoxide (DMSO, Sigma-Aldrich $(\circledR)$ was added and cultured for 10 minutes at $37^{\circ} \mathrm{C}$, and absorbance was measured in $570 \mathrm{~nm}$ wavelength.

\section{Statistical analysis}

SPSS v.21 statistics software was used. The Mann-Whitney test was used to compare the duplication times between flaps and control group. A p value $<0.05$ was considered significant.

\section{Results}

All the samples, either obtained from cartilage flaps (Group A) or head neck junction (Group B), showed chondrocyte viability. The average cellular viability of our samples was $43 \%$ (SD:8.5; range 30-60), there was no difference between chondrocyte viability between groups.
The mean duplication time was $58.5+19.5$ hours for chondral flaps samples and $58+15.1$ hours for the control group. There was no significant difference between them $(p=0.85)$

\section{Discussion}

Chondral flap forms part of a spectrum of cartilage damage ranging from loss of fixation to subchondral bone (ie wave sign) to exposed bone. Cartilage damage secondary to FAI has been a matter of concern as it was identified as a poor prognostic factor [13]. The possibility to preserve native cartilage would be preferable over other techniques like micro fractures with formation of fibrocartilage, however in order to be successful with cartilage preservation techniques the cartilage flap needs to be viable and able to heal. Our study adds some basic science information about cartilage flap viability and the ability of its chondrocytes to duplicate in an in vitro setting.

Previous studies have reported the viability of chondral flap type injuries in FAI population. Meulenkamp et al in reported cell viability and cartilage histologic characteristics in 12 flaps excised from 11 patients. Under light microscopy a musculoskeletal pathologist assessed the cartilage viability and found more than $90 \%$ of viable chondrocytes in 11 out of 12 samples. In addition, they found that in $50 \%$ of the flaps the cartilage was predominantly hyaline [14].

Hariri et al analyzed 10 flaps from 10 patients obtained during hip arthroscopy due to FAI. They assessed cellular viability with an automated system and found a mean cellular viability of $39 \%$. Furthermore, they found with biochemical analysis lower chondrocyte content, collagen content and chondrocyte biosynthesis as compared with reference values obtained from animal models [15].

As opposed to Meulenkamp's results our study showed a mean cellular viability of $43 \%$, similar to the value reported by Hariri et al. We believe this may be in part due to the method used in Meulenkamp's study in which the pathologist, based on cellular characteristics, assessed viability. Also in Meulenkamp's study the cartilage flap samples were obtained by surgical dislocation instead of hip arthroscopy.

In our study all the chondrocytes obtained from cartilage samples were able to duplicate, moreover there was no difference between the flaps and the control group. We believe that having studied the ability of the chondrocytes to duplicate in an in vitro setting adds more information about the capabilities of the cells present in the flap and their potential. We are not aware of previous studies reporting this finding and we did not find reference values for duplication times of hip chondrocytes.

We believe current evidence supports cellular viability in the chondral flap type injuries found in FAI. Although it has been shown that viability is not optimal and it has a poor biochemical environment [15], we think it may act as a scaffold providing mechanical support and be a better option than the fibrocartilage obtained after microfracture. Good early clinical results have been reported with repair techniques, however, this results need to be interpreted carefully as they could be due to repair 
techniques or as a result of concomitant traditional arthroscopic procedures done in FAI surgery.

Our study has some limitations. First, there was no macroscopic or histologic evaluation prior to the assessment of the samples. It would have been interesting to assess if the intraoperative appearance and/or histologic characteristics such as the percentage of fibrocartilage have a correlation with the percentage of viable cells or the duplication time. Second, we did not do an immunohistochemical characterization of the cells prior culture them. Therefore, we cannot be sure whether the cultured cells were only chondrocytes or if there was some contribution from fibroblasts.

As a conclusion, there is evidence to support cellular viability of the chondral flap in FAI surgery however, we believe more clinical studies need to be done to determine if chondral flap repair offers any advantage over other treatment options.

\section{References}

1. Ganz R, Parvizi J, Beck M, Leunig M, Nötzli H, Siebenrock KA. Femoroacetabular impingement: A cause for osteoarthritis of the hip. Clin Orthop. 2003;417:112-120. doi: 10.1097/01. blo.0000096804.78689.c2

2. Egerton T, Hinman RS, Takla A, Bennell KL, O’Donnell J. Intraoperative cartilage degeneration predicts outcome 12 months after hip arthroscopy. Clin Orthop Relat Res. 2013;471(2):593-599. doi:10.1007/s11999-012-2594-y

3. Nepple JJ, Larson CM, Smith MV, Kim YJ, Zaltz I, Sierra RJ, et al The reliability of arthroscopic classification of acetabular rim labrochondral disease. The American Journal of Sports Medicine. 2012;40(10):2224-2229. doi:10.1177/0363546512457157

4. ClohisyJC,StJohn LC, Schutz AL. Surgical treatment of femoroacetabular impingement: a systematic review of the literature. Clin Orthop Relat Res. 2010;468(2):555-564. doi: 10.1007/s11999-009-1138-6

5. Sampson TG. Arthroscopic treatment for chondral lesions of the hip. Clin Sports Med. 2011;30(2):331-348. doi: 10.1016/j.csm.2010.12.012

6. Bitar El YF, Lindner D, Jackson TJ, Domb BG. Joint-preserving surgical options for management of chondral injuries of the hip. J Am Acad Orthop Surg. 2014;22(1):46-56. doi: 10.5435/JAAOS-22-01-46

7. Sankar WN, Matheney TH, Zaltz I. Femoroacetabular impingement: Current concepts and controversies. Orthop Clin North Am. 2013;44(4):575-589. doi: 10.1016/j.ocl.2013.07.003

8. Konan S, Rayan F, Meermans G, Witt J, Haddad FS. Validation of the classification system for acetabular chondral lesions identified at arthroscopy in patients with femoroacetabular impingement. J Bone Joint Surg Br. 2011;93(3):332-336. doi: 10.1302/0301-620X.93B3.25322

9. Amenabar T, Piriz J, Mella C, Hetaimish BM, O’Donnell J. Reliability of 3 Different Arthroscopic Classifications for Chondral Damage of the Acetabulum. Arthroscopy. 2015;31(8):1492-1496. doi: 10.1016/j. arthro.2015.02.029

10. Stafford GH, Bunn JR, Villar RN. Arthroscopic repair of delaminated acetabular articular cartilage using fibrin adhesive: Results at one to three years. Hip Int. 2011;21(6):744-750. doi: 10.5301/ HIP.2011.8843

11. Fontana A, Bistolfi A, Crova M, Rosso F, Massazza G. Arthroscopic treatment of hip chondral defects: Autologous chondrocyte transplantation versus simple debridement. A pilot study. Arthroscopy. 2012;28(3):322-329. doi: 10.1016/j.arthro.2011.08.304

12. Jordan MA, Van Thiel GS, Chahal J, Nho SJ. Operative treatment of chondral defects in the hip joint: A systematic review. Curr Rev Musculoskelet Med. 2012;5(3):244-253. doi: 10.1007/s12178-0129134-y

13. Peters CL, Erickson JA. Treatment of femoro-acetabular impingement with surgical dislocation and débridement in young adults.J Bone Joint Surg Am. 2006;88(8):1735-1741. doi: 10.2106/JBJS.E.00514

14. Meulenkamp B, Gravel D, Beaulé PE. Viability assessment of the chondral flap in patients with cam-type femoroacetabular impingement: a preliminary report. Can J Surg. 2014;57(1):44-48. doi:10.1503/cjs.003513

15. Hariri S, Truntzer J, Smith RL, Safran MR. Biochemical and Cellular Assessment of Acetabular Chondral Flaps Identified During Hip Arthroscopy. Arthroscopy. 2015;31(6):1077-1083. doi: 10.1016/j. arthro.2015.01.010 